\title{
Localized Topology Control for Heterogeneous Wireless Ad-hoc Networks
}

\author{
Xiang-Yang $\mathrm{Li}^{\star} \quad$ Wen-Zhan Song ${ }^{\star}$ Yu Wang ${ }^{\dagger}$
}

\begin{abstract}
We study topology control in heterogeneous wireless ad hoc networks, where mobile hosts may have different maximum transmission powers and two nodes are connected iff they are within the maximum transmission range of each other. We present several strategies that all wireless nodes self-maintain sparse and power efficient topologies in heterogeneous network environment with low communication cost. The first structure is sparse and can be used for broadcasting. While the second structure keeps the minimum power consumption path, and the third structure is a length and power spanner with a bounded degree. Both the second and third structures are power efficient and can be used for unicast. Here a structure is power efficient if the total power consumption of the least cost path connecting any two nodes in it is no more than a small constant factor of that in the original heterogeneous communication graph. All our methods use at most $O(n)$ total messages, where each message has $O(\log n)$ bits.
\end{abstract}

Keywords-Graph theory, wireless ad hoc networks, topology control, heterogeneous networks, power consumption.

\section{INTRODUCTION}

An important requirement of wireless ad hoc networks is that they should be self-organizing, i.e., transmission ranges and data paths are dynamically restructured with changing topology. Localized ad hoc network topology control scheme is to let each wireless node locally adjust its transmission power and select proper neighbors to communicate according to certain strategy, while maintaining a structure that can support energy efficient routing and improve the overall network performance. Hence it can efficiently conserve the transmission energy from soft aspects with low cost. In the past several years, topology control algorithms have drawn significant research interest. Centralized algorithms can achieve optimality or its approximation, which are more applicable to static networks due to the lack of adaptability to topology changes. In contrast, distributed algorithms are more suitable for mobile ad hoc networks since the environment is inherently dynamic and they are adaptive to topology changes at the cost of possible less optimality. Furthermore, these algorithms only attempt to selectively choose some neighbors for each node. The primary distributed topology control algorithms for ad hoc networks aim to maintain network connectivity, optimize network throughput with power-efficient routing, conserve energy and increase the fault tolerance.

Most prior art [1], [2], [3], [4], [5], [6] on network topology control assumed that wireless ad hoc networks are modelled by unit disk graphs (UDG), i.e., two mobile hosts can communicate as long as their Euclidean distance is no more than a threshold. However, practically, wireless ad hoc networks cannot be perfectly modelled as UDGs: the maximum transmission ranges of wireless devices may vary due to various reasons such as the device differences and the small mechanic/electronic errors dur-

\footnotetext{
${ }^{\star}$ Department of Computer Science, Illinois Institute of Technology. Email: xli@cs.iit.edu, songwendit.edu. The work of Xiang-Yang Li is partially supported by NSF CCR-0311174.

${ }^{\dagger}$ Department of Computer Science, University of North Carolina at Charlotte.

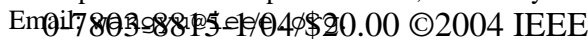

ing the process of transmitting even the transmission powers of all devices are set the same initially. In [7], [8], the authors extended UDG into a new model, called quasi unit disk graphs, which is closer to reality than UDG. In this paper, we study a more generalized model. Each wireless node $u$ may have its own transmission radius $r_{u}$. Then heterogeneous wireless networks are modelled by mutual inclusion graphs (MG): two nodes can communicate directly only if they are within the transmission range of each other, i.e., it has a link $u v$ iff $\|u v\| \leq \min \left(r_{u}, r_{v}\right)$. Clearly UDG is a special case of MG. Few research efforts addressed the topology control for heterogeneous wireless networks.

The main contribution of this paper is as follows. We propose several localized strategies for heterogeneous wireless devices to self-form a globally sparse power efficient network topology: a power spanner, a sparse structure and a degree-bounded length and power spanner respectively. Here an algorithm is said to construct a topology $H$ locally, if every node $u$ can decide which incident edge $u v$ belong to $H$ using only the information of nodes within a constant number of hops of $u$. All our algorithms have communication costs $O(n)$, where each message has $O(\log n)$ bits. Notice, to study the topology control in heterogeneous networks, it would be helpful to extend the ideas from the well-studied topologies, such as GG, RNG and Yao, used in homogeneous networks. The topology control for heterogeneous networks is not trivial, since many properties in homogeneous networks disappear in heterogeneous networks.

The rest of the paper is organized as follows. In Section II we introduce the background and review previous methods. Limitations on heterogeneous network topology control are discussed in Section III. We describe a strategy for all nodes forming a sparse structure in Section IV, a sparse power spanner in Section $\mathrm{V}$, and a degree-bounded power and length spanner in Section VI. We also analyze the communication complexities of these methods. Our theoretical results are corroborated in the simulations in Section VII. We conclude our paper in Section VIII with the discussion of future works.

\section{PRELIMINARIES}

\section{A. Heterogeneous Wireless Network Model}

A heterogeneous wireless ad hoc network is composed of a set $V$ of $n$ nodes $v_{1}, v_{2}, \cdots, v_{n}$, in which each node $v_{i}$ has its own maximum transmission power $p_{i}^{\prime}$. Let $\epsilon_{i}$ be the mechanic/electronic error of a node $v_{i}$ in its power control. Then the maximum transmission power considered in this paper is actually $p_{i}=p_{i}^{\prime}-\epsilon$. We adopt a common assumption in the literature that the power needed to support the communication between two nodes $v_{i}$ and $v_{j}$ is $\left\|v_{i} v_{j}\right\|^{\beta}$, where $\beta \in[2,5]$ is

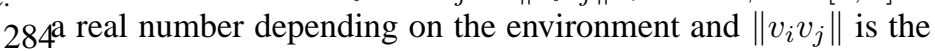


Euclidean distance between $v_{i}$ and $v_{j}$. Consequently, the signal sent by a node $v_{i}$ can be received by all nodes $v_{j}$ with $\left\|v_{i} v_{j}\right\| \leq r_{i}$, where $r_{i}^{\beta} \leq p_{i} / p_{0}, p_{0}$ is the uniform threshold that a signal with power $p_{0}$ can be recognized by a node. Thus, for simplicity, we assume that each mobile host $v_{i}$ has its own transmission range $r_{i}$. The heterogeneous wireless ad hoc network is then modelled by a mutual inclusion graph (MG), where two nodes $v_{i}, v_{j}$ are connected iff they are within the transmission range of each other, i.e., $\left\|v_{i} v_{j}\right\| \leq \min \left(r_{i}, r_{j}\right)$. Previously, no method is known for topology control when the networks are modelled as mutual inclusion graphs.

\section{B. Current State of Knowledge}

Many structures were proposed for topology control in homogeneous wireless ad hoc networks. Due to limited spaces, we will briefly review some of proximity geometric structures. The relative neighborhood graph [9] $R N G(V)$ consists of all edges $u v$ such that the intersection of two circles centered at $u$ and $v$ and with radius $\|u v\|$ do not contain any vertex $w$ from $V$. The Gabriel graph [10] $G G(V)$ contains edge $u v$ if and only if $\operatorname{disk}(u, v)$ contains no other points of $S$, where $\operatorname{disk}(u, v)$ is the disk with edge $u v$ as a diameter. Both $G G(V)$ and $R N G(V)$ are connected, planar, and contain the Euclidean minimum spanning tree of $V$. The intersections of $G G(V), R N G(V)$ with a connected $U D G(V)$ are connected. Delaunay triangulation, denoted by $\operatorname{Del}(V)$, is also used as underlying structure by several routing protocols. Here a triangle $\triangle u v w$ belongs to $\operatorname{Del}(V)$ if its circumcircle does not contain any node inside. It is well known that $R N G(V) \subseteq G G(V) \subseteq \operatorname{Del}(V)$. The intersection of $\operatorname{Del}(V)$ with a connected $U D G(V)$ has a bounded length spanning ratio [11].

The Yao graph [12] with an integer parameter $k \geq 6$, denoted by $\overrightarrow{Y G}_{k}(V)$, is defined as follows. At each node $u$, any $k$ equalseparated rays originated at $u$ define $k$ cones. In each cone, choose the shortest edge $u v$ among all edges from $u$, if there is any, and add a directed link $\overrightarrow{u v}$. Ties are broken arbitrarily or by ID. The resulting directed graph is called the Yao graph. Let $Y G_{k}(V)$ be the undirected graph by ignoring the direction of each link in $\overrightarrow{Y G}_{k}(V)$. Some researchers used a similar construction named $\theta$-graph [13], the difference is that it chooses the edge which has the shortest projection on the axis of each cone instead of the shortest edge in each cone.

The first effort for topology control in heterogeneous wireless networks was reported in [14] by the same authors of this paper. In [14], we showed how to perform topology control based on Yao structure for heterogeneous wireless networks. The results presented in current paper have been available online since around June 2003. Recently, several structures that extend the relative neighborhood graph and local minimum spanning tree were proposed in [15] for topology control in heterogeneous wireless networks. They build directed network topologies while the methods presented here build undirected topologies that are beneficial for routing. In addition, as the authors of [15] acknowledged, their original methods cannot guarantee the network connectivity. Then new methods were proposed to remedy this in their online version of the paper. Two structures were proposed by them: an extended relative neighborhood graph and 28 the extended local minimum spanning tree. It is unknown if their structures are sparse, power efficient.

\section{Spanners and Stretch Factors}

When constructing a subgraph of the original communication graph MG, we may need consume more power to connect some nodes since we may disconnect the most power efficient path in MG. Thus, naturally, we would require that the constructed structure approximates MG well in terms of the power consumption for unicast routing. In graph theoretical term, the structure should be a spanner [16], [13]. Let $G=(V, E)$ be a $n$-vertex weighted connected graph. The distance in $G$ between two vertices $u, v \in V$ is the length of the shortest path between $u$ and $v$ and it is denoted by $d_{G}(u, v)$. A subgraph $H=\left(V, E^{\prime}\right)$, where $E^{\prime} \subseteq E$, is a $t$-spanner of $G$ if for every $u, v \in V$, $d_{H}(u, v) \leq t \cdot d_{G}(u, v)$. The value of $t$ is called the stretch factor or spanning ratio. When the graph is a geometric graph and the weight is the Euclidean distance between two vertices, the stretch factor $t$ is called the length stretch factor, denoted by $\ell_{H}(G)$. For wireless networks, the mobile devices are usually powered by battery only. We thus pay more attention to the power consumptions. When the weight of a link $u v \in G$ is defined as the power to support the communication of link $u v$, the stretch factor of $H$ is called the power stretch factor, denoted by $\rho_{H}(G)$ hereafter. The power, denoted by $p_{G}(u, v)$, needed to support the communication between a link $u v$ in $G$ is often assumed to be $\|u v\|^{\beta}$, where $2 \leq \beta \leq 5$. Obviously, for any weighted graph $G$ and a subgraph $H \subseteq G$,

Lemma 1: [3] Graph $H$ has stretch factor $\delta$ if and only if for any link $u v \in G, d_{H}(u, v) \leq \delta \cdot d_{G}(u, v)$.

Thus, to generate a spanner $H$, we only have to make sure that every link of $G$ is approximated within a constant factor.

\section{Sparseness and Bounded Degree}

All well-known proximity graphs $(G G(V), \quad R N G(V)$, $\operatorname{Del}(V)$ and $Y G(V)$ ) have been proved to be sparse graphs when network is modeled as a UDG. Recall that a sparse graph means the number of edges is linear with the number of nodes. The sparseness of all well-known proximity graphs implies that the average node degree is bounded by a constant. Moreover, we prefer the maximum node degree is bounded by a constant, because wireless nodes have limited resources and the signal interference in wireless communications. Unbounded degree (or indegree) at a node $u$ will often cause large overhead at $u$, whereas a bounded degree increases the network throughput. In addition, bounded degree will also give us advantages when apply several routing algorithms. Therefore, it is often imperative to construct a sparse network topology with a bounded node degree while it is still power-efficient. However, Li et al. [3] showed that the maximum node degree of RNG, GG and Yao could be as large as $n-1$. The instance consists of $n-1$ points lying on the unit circle centered at a node $u \in V$. Then each edge $u v_{i}$ belongs to the $R N G(V), G G(V)$ and $\overrightarrow{Y G}_{k}(V)$.

Recently, in homogeneous wireless ad hoc networks, some improved or combined proximity graphs [17], [18] have been proposed to build planar degree-bounded power spanner topology, which meets all preferred properties for unicast. In het-

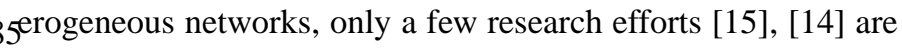


reported so far. In the following, we will first discuss the difficulties and limitations for topology control in heterogeneous networks, then present our localized strategies in detail.

\section{LIMITATIONS}

In heterogeneous wireless ad hoc networks, the planar topology does not necessarily exist. Figure 1 (a) shows an example, there are four nodes $x, y, u$ and $v$ in the network, where their transmission range $r_{x}=r_{y}=\|x y\|$ and $r_{u}=r_{v}=\|u v\|$, and node $u$ is out of the transmission range of node $x$ and $y$, while node $v$ is in the transmission range of node $y$ and out of the range of $x$. The transmission ranges of $x$ and $y$ are illustrated by the dotted circles. According to the definition of $M G$, there are only three edges $x y, v y$ and $u v$ in the graph. Hence any topology control method can not make the topology planar while keeping the communication graph connected. On the other hand, it is worth to think whether we can design a new routing protocol on some pseudo-planar topologies. As will see later, the pseudo-planar topology GG(MG) and RNG(MG) proposed in this section has some special properties which are different from other general non-planar topologies. For instance, two intersecting triangles can not share a common edge. We leave it as a future work.

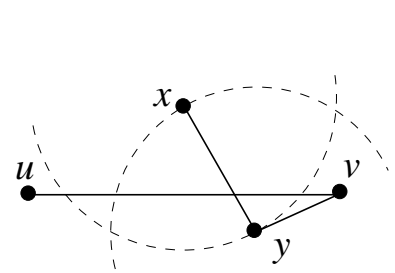

(a)

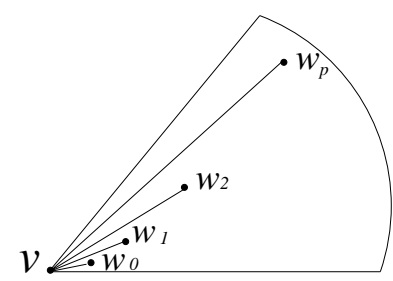

(b)
Fig. 1. Limitations on heterogeneous networks: (a) Planar topology does not exist. (b) Degree of node $v$ can not be bounded by constant.

We also can show that the node degree in heterogeneous networks can not be bounded by a constant if the radius ratio is unbounded. Figure 1 (b) shows such an example. In the example, a node $v$ has $p+1$ incoming neighbors $w_{i}, 0 \leq i \leq p$. Assume that each node $w_{i}$ has a transmission radius $r_{w_{i}}=r_{v} / 3^{p-i}$ and $\left\|v w_{i}\right\|=r_{w_{i}}$. Obviously, $\left\|w_{i} w_{j}\right\|>\min \left(r_{w_{i}}, r_{w_{j}}\right)$, i.e., any two nodes $w_{i}, w_{j}$ are not directly connected in MG.

Obviously, none of those edges incident on $v$ can be deleted, hence there is no topology control method to bound the degree by a constant without violating connectivity. Consider the example illustrated by Figure 1 (b), edges $v w_{i}, 0 \leq i \leq p$, are all possible communication links. Thus, node $v$ in any connected spanning graph has degree $p+1$. On the other hand, the topology generated by our method in section VI can gurantee the maximum node degree bounded by $O\left(\log _{2} \gamma\right)$, where

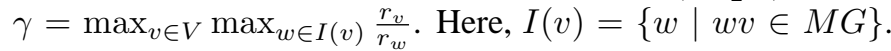
This is optimal in the worst case. In previous example, recall that $3^{p} r_{w_{0}}=r_{v}$, hence $\gamma$ equals to $3^{p}$. Thus, $v$ has degree $\log _{3} \gamma+1=\Theta\left(\log _{2} \gamma\right)$. In the paper, we always assume $\gamma$ is a constant. It is practical, since it is trivial that two wireless devices in same network have unbounded radius ratio.

\section{Heterogeneous Sparse Structure}

In this section, we propose a strategy for all nodes to selfform a sparse structure, called $R N G(M G)$, based on the rela 28 tive neighborhood graph structure, whose total number of links is $O(n)$. We add a link $u v \in M G$ to $R N G(M G)$ if there is $n o$ another node $w$ inside lune $(u, v)$ and both links $u w$ and $w v$ are in MG. Here lune $(u, v)$ is the intersection of $\operatorname{disk}(u,\|u v\|)$ and $\operatorname{disk}(v,\|u v\|)$. The algorithm will be similar to Algorithm 2 , thus we omit it here. Notice that the total communication cost of constructing $R N G(M G)$ is $O(n \log n)$ bits, assuming that the radius and ID information of a node can be represented in $O(\log n)$ bits. In addition, the structure $R N G(M G)$ is symmetric: if a node $u$ keeps a link $u v$, node $v$ will also keep the link $u v$. Thus, a node $u$ does not have to tell its neighbor $v$ whether it keeps a link $u v$ or not.

It is not difficult to prove that structure $R N G(M G)$ is connected by induction. On the other hand, same as the case in homogeneous networks (i.e., UDG mode), $R N G(M G)$ does not have a bounded length stretch factor, nor constant bounded power stretch factor, and does not have bounded node degree. In this paper, we will show that $R N G(M G)$ is a sparse graph: it has at most $6 n$ links.

In the following, we define a new structure, called

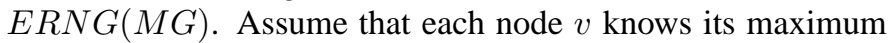
transmission radius $r_{v}$. Let $B(u)=\left\{v \mid r_{v} \geq r_{u}\right\}$. A node $u$ processes its incident link $u v$ in MG only if $r_{v} \geq r_{u}$, i.e., $v \in B(u)$. Node $u$ removes a link $u v$, where $v \in B(u)$, if there is another node $w \in B(u)$ inside lune $(u, v)$ with both links $u w$ and $w v$ are in MG. All the links $u v$ kept by all nodes form the final structure $E R N G(M G)$.

\section{Algorithm 1: Constructing-ERNG}

1. Each node $u$ initiates sets $E_{M G}(u)$ and $E_{E R N G}(u)$ to be empty. Here $E_{M G}(u)$ is the set of links of MG known to $u$ so far and $E_{E R N G}(u)$ is the set of links of ERNG known to $u$ so far. Then, each node $u$ locally broadcasts a HELLO message with $I D_{u}, r_{u}$ and its position $\left(x_{u}, y_{u}\right)$ to all nodes in its transmission range. Note that $r_{u}=p_{u}{ }^{1 / \beta}$ is its maximum transmission range. 2 . At the same time, each node $u$ processes the incoming messages. Assume that node $u$ gets a message from some node $v$. If $\|v u\| \leq \min \left\{r_{u}, r_{v}\right\}$, then node $u$ adds a link $u v$ to $E_{M G}(u)$. If $r_{v} \geq r_{u}$, then node $u$ performs the following procedures. Node $u$ checks if there is another link $u w \in E_{M G}(u)$ with the following additional properties: 1) $w \in$ lune $(u, v), 2) r_{w} \geq r_{u}$, and 3) $\|w v\| \leq \min \left\{r_{w}, r_{v}\right\}$. If no such link $u w$, then add $u v$ to $E_{E R N G}(u)$. For any link $u w \in E_{E R N G}(u)$, node $u$ checks if the following conditions hold: 1) $v \in \operatorname{lune}(u, w)$, and 2) $\|w v\| \leq \min \left\{r_{w}, r_{v}\right\}$. If the conditions hold, then remove link $u w$ from $E_{E R N G}(u)$.

3. Node $u$ repeats the above steps until no new Hello messages received.

4. For each link $u v \in E_{E R N G}(u)$, node $u$ informs node $v$ to add link $u v$.

5. All links $u v$ in $E_{E R N G}(u)$ are the final links in $\operatorname{ERNG}(M G)$ incident on $u$.

We then prove that the structure ERNG has at most $6 n$ links.

Lemma 2: Structure ERNG(MG) has at most $6 n$ links.

Proof: Consider any node $u$. We will show that $u$ keeps at most 6 directed links emanated from $u$. Assume that $u$ keeps more than 6 directed links. Obviously, there are two links $u w$ and $u v$ such that $\angle w u v<\pi / 3$. Thus, $v w$ is not the longest link $86^{\text {in triangle }} \triangle u v w$. Without loss of generality, we assume that 
$\|u w\|$ is the longest in triangle $\triangle u v w$. Notice that the existence of link $u w$ implies that $\|u w\| \leq \min \left(r_{u}, r_{w}\right)=r_{u}$. Consequently, $\|v w\| \leq\|u w\| \leq \min \left(r_{u}, r_{w}\right)$. Thus, from the fact that $r_{u} \leq r_{v}$, we know $\|v w\| \leq \min \left(r_{v}, r_{w}\right)$. Hence, link $v w$ does exist in the original communication graph MG, it implies that link $u w$ cannot be selected to ERNG.

From Lemma 2, we can prove the following lemma.

Lemma 3: Structure RNG(MG) has at most $6 n$ links.

Proof: Imagine that each link $u v$ has a direction as follows: $\overrightarrow{u v}$ if $r_{u} \leq r_{v}$. Then similar to Lemma 2, we can prove that each node $u$ only keeps at most 6 such imagined direct links. Thus, total links are at most $6 n$.

Similarly, we can define a structure $E G G(M G)$, which contains an edge $u v$ if $r_{u} \leq r_{v}$ and there is no node $w$ with the following properties: 1) $\left.r_{u} \leq r_{w}, 2\right) w$ is inside the disk $\operatorname{disk}(u, v)$. However, we cannot prove that $E G G(M G)$ has a linear number of links.

\section{Heterogeneous Power Spanner}

Then, we give a strategy for all nodes to self-form a power spanner structure, called $G G(M G)$, based on the Gabriel graph. We add a link $u v \in M G$ to $G G(M G)$ if there is no another node $w$ inside $\operatorname{disk}(u, v)$ and both links $u w$ and $w v$ are in MG. Our localized construction method works as follows.

\section{Algorithm 2: Constructing-GG}

1. Let $E_{M G}(u)$ and $E_{G G}(u)$ are the set of links known to $u$ from MG and $G G$ respectively. Each node $u$ initiates both $E_{M G}(u)$ and $E_{G G}(u)$ as empty. Then, each node $u$ locally broadcasts a HELLO message with $I D_{u}, r_{u}$ and its position $\left(x_{u}, y_{u}\right)$ to all nodes in its transmission range.

2. At the same time, each node $u$ processes the incoming messages. Assume that node $u$ gets a message from some node $v$. If $\|v u\| \leq \min \left\{r_{u}, r_{v}\right\}$, then node $u$ adds a link $u v$ to $E_{M G}(u)$. Node $u$ checks if there is another link $u w \in E_{M G}(u)$ with the following two additional properties: 1) $w \in \operatorname{disk}(u, v)$, and 2) $\|w v\| \leq \min \left\{r_{w}, r_{v}\right\}$. If no such link $u w$, add $u v$ to $E_{G G}(u)$. For any link $u w \in E_{G G}(u)$, node $u$ checks if the following two properties hold: 1) $v \in \operatorname{disk}(u, w)$, and 2) $\|w v\| \leq \min \left\{r_{w}, r_{v}\right\}$. If they hold, remove link $u w$ from $E_{G G}(u)$.

3. Node $u$ repeats the above steps until no new HELlO messages received.

4. All links $u v$ in $E_{G G}(u)$ are the final links in $G G(M G)$ incident on $u$.

We first show that Algorithm 2 builds the structure $G G(M G)$ correctly. For any link $u v \in G G(M G)$, clearly, we cannot remove them in Algorithm 2. For a link $u v \notin G G(M G)$, assume that a node $w$ is inside $\operatorname{disk}(u, v)$ and both links $u w$ and $w v$ belong to MG. If node $u$ gets the message from $w$ first, and then gets message from $v$, clearly, $u v$ cannot be added to $E_{G G}(u)$. If node $u$ gets the message from $v$ first, then $u$ will remove $u v$ from $E_{G G}(u)$ (if it is there) when $u$ gets the information of $w$.

It is not difficult to prove that structure $G G(M G)$ is connected by induction. In addition, since we remove a link $u v$ only if there are two links $u w$ and $w v$ with $w$ inside $\operatorname{disk}(u, v)$, it is easy to show that the power stretch factor of $G G(M G)$ is 1 . In other words, the minimum power consumption path for any 28 two nodes $v_{i}$ and $v_{j}$ in MG is still kept in $G G(M G)$. Remember that here we assume the power needed to support a link $u v$ is $\|u v\|^{\beta}$, for $\beta \in[2,5]$.

On the other hand, same as the case in homogeneous networks (i.e., UDG mode), $G G(M G)$ is not a length spanner, and does not have bounded node degree. Furthermore, it is unknown whether $G G(M G)$ is a sparse graph. Recently, it was proven in [19] that $G G(M G)$ has at most $O\left(n^{8 / 5} \log \gamma\right)$ edges where $\gamma=\max r_{u} / r_{v}$.

Notice that, the extension from Gabriel graph is non-trivial. In [19], two structures defined as follows even cannot guarantee the connectivity. In the first structure, called $L G G_{0}(M G)$, they remove a link $u v \in M G$ if there is another node $w$ inside $\operatorname{disk}(u, v)$. In the second structure, called $L G G_{1}(M G)$, they remove a link $u v \in M G$ if there is another node $w$ inside $\operatorname{disk}(u, v)$, and either link $u w$ or link $w v$ is in MG.

\section{Heterogeneous DegReE-Bounded SPANNER}

Undoubtedly, as described in preliminaries, we always prefer a structure has more nice properties, such as degree-bounded (stronger than sparse), power spanner etc. Naturally, we could extend the previous known degree-bounded spanner, such as the Yao related structures, from homogeneous networks to heterogeneous networks. Unfortunately, a simple extension of the Yao structure from UDG to MG even does not guarantee the connectivity. Figure 2 (a) illustrates such an example. Here $r_{u}=r_{v}=\|u v\|, r_{w}=\|u w\|, r_{x}=\|v x\|$, and $\|u w\|<\|u v\|$, $\|u w\|<\|v w\|,\|v x\|<\|u v\|$, and $\|v x\|<\|u x\|$. In addition, $v$ and $w$ are in the same cone of node $u$, and nodes $x$ and $u$ are in the same cone of node $v$. Thus, the original MG graph contains links $u v, u w$ and $v x$ only and is connected. However, when applying Yao structure on all nodes, node $u$ will only have information of node $v$ and $w$ and it will keep link $u w$. Similarly, node $w$ keeps link $u w$; node $v$ keeps link $v x$; and node $x$ keeps link $x v$. In other words, only link $x v$ and $u w$ are kept by Yao method. Thus applying Yao structure disconnects node $v, x$ from the other two nodes $u$ and $w$. Consequently, we need more sophisticated extensions of the Yao structure to MG to guarantee the connectivity of the structure.

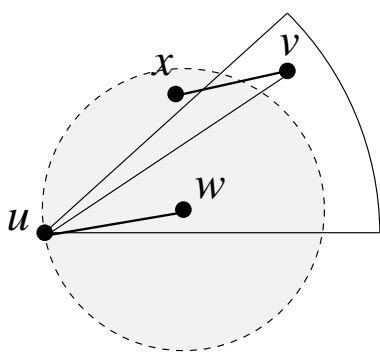

(a)

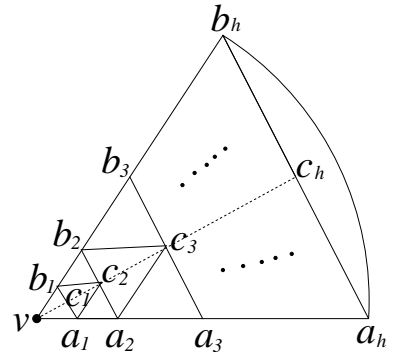

(b)
Fig. 2. Extend Yao structure on heterogeneous networks: (a) Simple extension of Yao structure does not guarantee the connectivity. (b) Further space partition in each cone to bound in-degree.

\section{A. Extended Yao Graph}

\section{Algorithm 3: Constructing-EYG}

1. Initially, each node $u$ divides the disk $\operatorname{disk}\left(u, r_{u}\right)$ centered f7at $u$ with radius $r_{u}$ by $k$ equal-sized cones centered at $u$. We 
generally assume that the cone is half open and half-close. Let $\mathbb{C}_{i}(u), 1 \leq i \leq k$, be the $k$ cones partitioned. Let $C_{i}(u), 1 \leq$ $i \leq k$, be the set of nodes $v$ inside the $i$ th cone $\mathbb{C}_{i}(u)$ with a larger or equal ${ }^{1}$ radius than $u$. In other words,

$$
C_{i}(u)=\left\{v \mid v \in \mathbb{C}_{i}(u), \text { and } r_{v} \geq r_{u}\right\} .
$$

Initially, $C_{i}(u)$ is empty.

2. Each node $u$ broadcasts a HELLO message with $I D_{u}, r_{u}$ and its position $\left(x_{u}, y_{u}\right)$ to all nodes in its transmission range.

3. At the same time, each node $u$ processes the incoming broadcast messages. Once it gets a HELLO message from some node $v$, it sets $C_{i}(u)=C_{i}(u) \bigcup\{v\}$, if node $v$ is inside the $i$ th cone $\mathbb{C}_{i}(u)$ of node $u$ and $r_{v} \geq r_{u}$.

4. Node $u$ chooses a node $v$ from each cone $C_{i}(u)$ so that the link $u v$ has the smallest $I D(u v)$ among all links $u v_{j}$ with $v_{j}$ in $C_{i}(u)$, if there is any.

5. Finally, each node $u$ informs all 1-hop neighbors of its chosen links through a broadcast message. Let $\overrightarrow{E Y G}_{k}(M G)$ be the union of all chosen links.

Since the symmetric communications are required, let $E Y G_{k}(M G)$ be the undirected graph by ignoring the direction of each link in $\overrightarrow{E Y G}_{k}(M G)$. Graph $E Y G_{k}(M G)$ is the final network topology. Since node $u$ chooses a node $v \in \operatorname{disk}\left(u, r_{u}\right)$ with $r_{v} \geq r_{u}$, link $u v$ is indeed a bidirectional link, i.e., $u$ and $v$ are within the transmission range of each other. Additionally, this strategy could avoid the possible disconnection by simple Yao extension we mentioned before.

Obviously, each node only broadcasts twice: one for broadcasting its ID, radius and position; and the other for broadcasting the selected neighbors. Remember that it selects at most $k$ neighbors. Thus, each node sends messages at most $O((k+1) \cdot \log n)$ bits. Here, we assume that the node ID and its position can be represented using $O(\log n)$ bits for a network with $n$ wireless nodes.

Before we study the properties of this structure, we have to define some terms first. Assume that each node $v_{i}$ of MG has a unique identification number $I D_{v_{i}}=i$. The identity of a bidirectional link $u v$ is defined as $I D(u v)=\left(\|u v\|, I D_{u}, I D_{v}\right)$ where $I D_{u}>I D_{v}$. Note that we use the bidirectional links instead of the directional links in the final topology to guarantee connectivity. In other words, we require that both node $u$ and node $v$ can communicate with each other through this link. In this paper, all proofs about connectivity or stretch factors take the notation $u v$ and $v u$ as same, which is meaningful. Only in the topology construction algorithm or proofs about boundeddegree, $u v$ is different than $v u$ : the former is initiated and built by $u$, whereas the latter is by node $v$. Sometimes we denote a directional link from $v$ to $u$ as $\overrightarrow{v u}$ if necessary. Then we can order all bidirectional links (at most $n(n-1)$ such links) in an increasing order of their identities. Here the identities of two links are ordered based on the following rule: $I D(u v)>I D(p q)$ if (1) $\|u v\|>\|p q\|$ or (2) $\|u v\|=\|p q\|$ and $I D_{u}>I D_{p}$ or (3) $\|u v\|=\|p q\|, u=p$ and $I D_{v}>I D_{q}$.

Correspondingly, the rank of each link $u v$, denoted by $\operatorname{rank}(u v)$, is its order in sorted bidirectional links. Notice that,

\footnotetext{
${ }^{1}$ This is the main difference between this algorithm and the simple extension of Yao structure discussed before, in which it considers all nodes $v$ that $u$ can get signal from.
}

we actually only have to consider the links in MG. We then show that the constructed network topology is a length and power spanner.

Theorem 4: The length stretch factor of $E Y G_{k}(M G), k>$ 6 , is at most $\ell=\frac{1}{1-2 \sin \left(\frac{\pi}{k}\right)}$.

Proof: Notice it is sufficient to show that for any nodes $u$ and $v$ with $\|u v\| \leq \min \left(r_{u}, r_{v}\right)$, i.e. $u v \in M G$, there is a path connecting $u$ and $v$ in $E Y G_{k}(M G)$ with length at most $\ell\|u v\|$. We construct a path $u \leftrightarrow v$ connecting $u$ and $v$ in $E Y G_{k}(M G)$ as follows.

Assume that $r_{u} \leq r_{v}$. If link $u v \in E Y G_{k}(M G)$, then set the path $u \leadsto v$ as the link $u v$. Otherwise, consider the $\operatorname{disk}\left(u, r_{u}\right)$ of node $u$. Clearly, node $u$ will get information of $v$ from $v$ and node $v$ will be selected to some $C_{i}(u)$ since $r_{v} \geq r_{u}$. Thus, from $u v \notin E Y G_{k}(M G)$, there must exist another node $w$ in the same cone as $v$, which is a neighbor of $u$ in $E Y G_{k}(M G)$. Then set $u \leadsto v$ as the concatenation of the link $u w$ and the path $w \leadsto v$. Here the existence of path $w \leadsto v$ can be easily proved by induction on the distance of two nodes. Notice that the angle $\theta$ of each cone section is $\frac{2 \pi}{k}$. When $k>6$, then $\theta<\frac{\pi}{3}$. It is easy to show that $\|w v\|<\|u v\|$. Consequently, the path $u \leadsto v$ is a simple path, i.e., each node appears at most once.

We then prove by induction that the path $u \leftrightarrow v$ has total length at most $\ell\|u v\|$.

Obviously, if there is only one edge in $u \leadsto v, d(u \leftrightarrow v)=$ $\|u v\|<\ell\|u v\|$. Assume that the claim is true for any path with $l$ edges. Then consider a path $u \leadsto v$ with $l+1$ edges, which is the concatenation of edge $u w$ and the path ${ }^{2} w \leftrightarrow v$ with $l$ edges, as shown in Figure 3 where $\|w v\|=\|x v\|$.

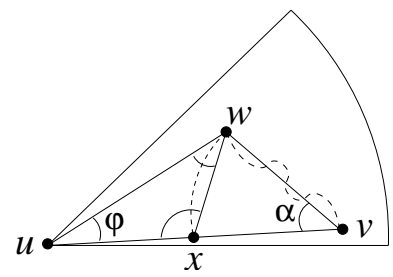

Fig. 3. The length stretch factor of $E Y G_{k}(M G)$ is at most $\frac{1}{1-2 \sin \left(\frac{\pi}{k}\right)}$.

Notice, from induction, $d(w \leftrightarrow v) \leq \ell\|w v\|$. Then, let $\varphi=\angle w u v$ and $\alpha=\angle u v w$, we have

$$
\begin{aligned}
\frac{\|u w\|}{\|u x\|} & =\frac{\sin (\angle u x w)}{\sin (\angle x w u)}=\frac{\sin \left(\frac{\pi}{2}+\frac{\alpha}{2}\right)}{\sin \left(\frac{\pi}{2}+\frac{\alpha}{2}+\varphi\right)} \\
& =\frac{1}{\cos \varphi-\sin \varphi \tan \frac{\alpha}{2}} \leq \frac{\cos \left(\frac{\pi}{4}-\frac{\varphi}{4}\right)}{\cos \left(\frac{\pi}{4}+\frac{3}{4} \varphi\right)} \leq \frac{1}{1-2 \sin \left(\frac{\pi}{k}\right)}
\end{aligned}
$$

The first inequality is because $0 \leq \alpha \leq \frac{\pi}{2}-\frac{\varphi}{2}$ and the second inequality is because $0 \leq \varphi \leq \frac{2 \pi}{k}$. Consequently, $d(u \leftrightarrow$ $v)=\|u w\|+d(w \leftrightarrow v)<\ell\|u x\|+\ell\|w v\|=\ell\|u v\|$, where $\ell=\frac{1}{1-2 \sin \left(\frac{\pi}{k}\right)}$. That is to say, the claim is also true for the path $u \leftrightarrow v$ with $l+1$ edges.

\footnotetext{
${ }^{2}$ In the procedure of induction, if $r_{w} \leq r_{v}$ then we induct on path $w \leftrightarrow r$, otherwise we induct on path $v \leadsto w$. In fact, here $w \leadsto v$ is same as $v \leadsto$ $w$ since the path is bidirectional for communication. Directional link is only considered in building process and is meaningless when we talk about the path.

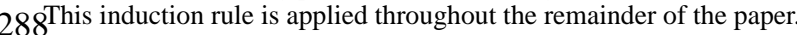


Thus, the length stretch factor of $E Y G_{k}(M G)$ is at most $\ell=$ $\frac{1}{1-2 \sin \left(\frac{\pi}{k}\right)}$. This finishes the proof.

Theorem 5: The power stretch factor of the extended Yao graph $E Y G_{k}(M G), k>6$, is at most $\rho=\frac{1}{1-\left(2 \sin \frac{\pi}{k}\right)^{\beta}}$.

Proof: The proof is similar to that in UDG [3], [4] except the induction procedure. We show by induction, on the number of its edges, that the path $u \leadsto v$ constructed in theorem 4 has power cost, denoted by $p(u \leftrightarrow v)$, at most $\rho\|u v\|^{\beta}$.

\section{B. Novel Space Partition}

Partitioning the space surrounding a node into $k$ equal-sized cones enables us to bound the node out-degree using the Yao structure. Using the same space partition, Yao-Yao structure [3], [4] produces a topology with bounded in-degree when the networks are modeled by UDG. Yao-Yao structure (for UDG) is generated as follows: a node $u$ collects all its incoming neighbors $v$ (i.e., $\overrightarrow{v u} \in \overrightarrow{Y G}_{k}(V)$ ), and then selects the closest neighbor $v$ in each cone $\mathbb{C}_{i}(u)$. Clearly, Yao-Yao has bounded degree at most $k$. They also showed that another structure YaoSink [3], [4] has not only the bounded node degree but also a constant bounded stretch factor. The network topology with a bounded degree can increase the communication efficiency. However, these methods [3], [4] may fail when the networks are modeled by MG: they cannot even guarantee the connectivity, which is verified by following discussions.

Assume that we already construct a connected directed structure $\overrightarrow{E Y G}_{k}(M G)$. Let $I(v)=\left\{w \mid \overrightarrow{w v} \in \overrightarrow{E Y G}_{k}(M G)\right\}$. In other words, $I(v)$ is the set of nodes that have directed links to $v$ in $\overrightarrow{E Y G}_{k}(M G)$. Let $I_{i}(v)=I(v) \cap \mathbb{C}_{i}(u)$, i.e., the nodes in $I(v)$ located inside the $i$ th cone $\mathbb{C}_{i}(v)$. Yao-Yao structures will pick the closest node $w$ in $I_{i}(v)$ and add undirected link $w v$ to Yao-Yao structure. Previous example in Figure 1 (b) also illustrates the situation that Yao-Yao structure is not connected. In the example, a node $v$ has $p+1$ incoming neighbors $w_{i}, 0 \leq i \leq p$. Assume that each node $w_{i}$ has a transmission radius $r_{w_{i}}=r_{v} / 3^{p-i}$ and $\left\|v w_{i}\right\|=r_{w_{i}}$. Obviously, $\left\|w_{i} w_{j}\right\|>\min \left(r_{w_{i}}, r_{w_{j}}\right)$, i.e., any two nodes $w_{i}, w_{j}$ are not directly connected in MG. It is easy to show that the Yao structure $\overrightarrow{E Y G}_{k}(M G)$ only has directed links $\overrightarrow{w_{i} v}$. Obviously, node $v$ will only select the closest neighbor $w_{0}$ to the Yao-Yao structure, which disconnects the network. This same example can also show that the structure based on Yao-Sink [3], [4] is also not connected for heterogeneous wireless ad hoc networks.

Thus, selecting the closest incoming neighbor in each cone $\mathbb{C}_{i}$ is too aggressive to guarantee the connectivity. Observe that, in Figure 1 (b), to guarantee the connectivity, when we delete a directed link $\overrightarrow{w_{i} v}$, we need to keep some link, say $w_{j} v$, such that $w_{i} w_{j}$ is a link in MG. Thus, we want to further partition the cone into a limited number of smaller regions and we will keep only one node in each region, e.g., the closest node. Clearly, to guarantee that other nodes in the same region are still connected to $v$, we have to make sure that any two nodes $w_{i}, w_{j} \in I(v)$ that co-exist in a same small region are directly connected in MG. Consequently, if the number of regions is bounded by a constant, a degree-bounded structure could be generated. In the remainder of this subsection, we will introduce a novel space partition strategy satisfying the above requirement.

\section{Method 1: Partition-EYG}

For each node $v$, let $\gamma_{v}=\max _{w \in I(v)} \frac{r_{v}}{r_{w}}$. Remember that all nodes in $I(v)$ have transmission radius at most $r_{v}$. Let $h$ be the positive integer satisfying $2^{h-2}<\gamma_{v} \leq 2^{h-1}$. We then discuss in detail our partition strategy of the cones, which is illustrated by Figure 2 (b). Each node $v$ divides each cone centered at $v$ into limited number of triangles and caps, where $\left\|v a_{i}\right\|=\left\|v b_{i}\right\|=$ $\frac{1}{2^{h-i}} r_{v}$ and $c_{i}$ is the mid-point of the segment $a_{i} b_{i}$, for $1 \leq$ $i \leq h$. Notice that this partition can be conducted by node $v$ locally since it can collect the transmission radius information of nodes in $I(v)$. The triangles $\triangle v a_{1} b_{1}, \triangle a_{i} b_{i} c_{i+1}, \triangle a_{i} a_{i+1} c_{i+1}$, $\triangle b_{i} b_{i+1} c_{i+1}$, for $1 \leq i \leq h-1$, and the cap $\widehat{a_{n} b_{n}}$ form the final space partition of each cone. For simplicity, we call such a triangle or the cap as a region. We then prove that this partition indeed guarantees that any two nodes in any same region are connected in MG.

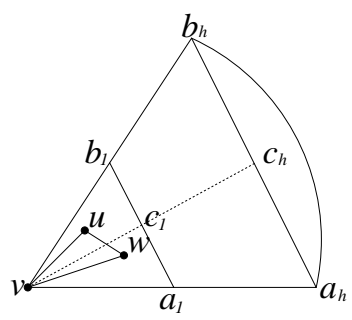

(a)

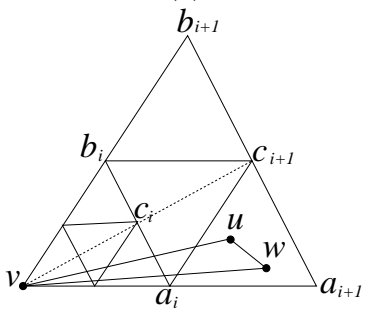

(c)

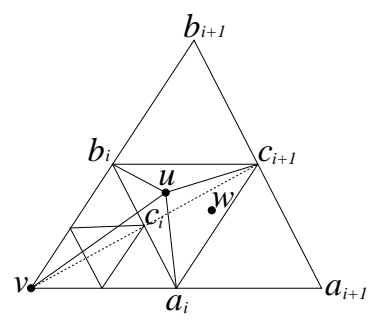

(b)

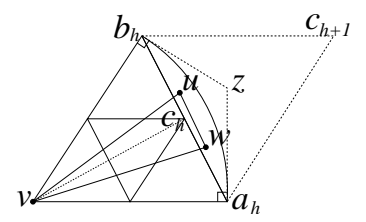

(d)
Fig. 4. (a) Two nodes are in triangle $\triangle v a_{1} b_{1}$. (b) Two nodes are in triangle $\triangle a_{i} b_{i} c_{i+1}$. (c) Two nodes are in triangle $\triangle a_{i} a_{i+1} c_{i+1}$. (d) Two nodes are inside cap $\widehat{a_{h} b_{h}}$.

Lemma 6: Assume that $k \geq 6$. Any two nodes $u, w \in I(v)$ that co-exist in any one of the generated regions are directly connected in $\mathrm{MG}$, i.e., $\|u w\|<\min \left(r_{u}, r_{w}\right)$.

Proof: There are four different cases.

1. Two nodes are in $\triangle v a_{1} b_{1}$, as shown in Figure 4 (a).

Remember that all nodes in $I(v)$ have transmission radius at least $\left\|v a_{1}\right\|=\frac{1}{2^{(h-1)}} r_{v}$. We have $\min \left(r_{u}, r_{w}\right) \geq\left\|v a_{1}\right\|=$ $\left\|v b_{1}\right\|$ and $\left\|a_{1} b_{1}\right\| \leq\left\|v a_{1}\right\|$. In addition, since $u w$ is a segment inside $\triangle v a_{1} b_{1}$, we have $\|u w\| \leq \max \left(\left\|a_{1} b_{1}\right\|,\left\|v a_{1}\right\|,\left\|v b_{1}\right\|\right)$. Consequently, $\|u w\|<\min \left(r_{u}, r_{w}\right)$, i.e. $u w \in M G$.

2. Two nodes are in $\triangle a_{i} b_{i} c_{i+1}$, as shown in Figure 4 (b).

In this case, we have

(a) $\|v u\|>\left\|u c_{i+1}\right\|$, since $a_{i} b_{i}$ is the perpendicular bisector of $v c_{i+1}$ and $u$ is at the same side of line $a_{i} b_{i}$ as $c_{i+1}$.

(b) $\|v u\|>\left\|u a_{i}\right\|$, because $\angle v a_{i} u>\frac{\pi}{3}>\angle u v a_{i}$.

(c) $\|v u\|>\left\|u b_{i}\right\|$, because $\angle v b_{i} u>\frac{\pi}{3}>\angle u v b_{i}$.

(d) $\|u w\|<\max \left(\left\|u c_{i+1}\right\|,\left\|u a_{i}\right\|,\left\|u b_{i}\right\|\right)$, because node $w$ must be inside one of the triangles $\triangle a_{i} b_{i} u, \triangle a_{i} c_{i+1} u$ and $\triangle b_{i} c_{i+1} u$. 289 Thus, $\|u w\|<\|u v\|$. Similarly, $\|u w\|<\|w v\|$. Consequently, 
$u w \in M G$ from

$$
\|u w\|<\min (\|u v\|,\|w v\|)<\min \left(r_{u}, r_{w}\right) .
$$

3. Two nodes are in $\triangle a_{i} a_{i+1} c_{i+1}$, as shown in Figure 4 (c). We have $\min \left(r_{u}, r_{w}\right) \geq\left\|v a_{i}\right\|=\left\|a_{i} a_{i+1}\right\|=\left\|a_{i} c_{i+1}\right\|>$ $\left\|a_{i+1} c_{i+1}\right\|$. Since $u w$ is a segment inside $\triangle a_{i} a_{i+1} c_{i+1}$, $\|u w\|<\max \left(\left\|a_{i} a_{i+1}\right\|,\left\|a_{i} c_{i+1}\right\|,\left\|a_{i+1} c_{i+1}\right\|\right) \leq \min \left(r_{u}, r_{w}\right)$, i.e. $u w \in M G$. Triangle $\triangle b_{i} b_{i+1} c_{i+1}$ is the symmetric case with triangle $\triangle a_{i} a_{i+1} c_{i+1}$, so the claim holds similarly.

4. Two nodes are inside the cap $\widehat{a_{h} b_{h}}$, as shown in Figure 4 (d), where $a_{h} z$ and $b_{h} z$ is the tangent of arc $\widehat{a_{h} b_{h}}$ at point $a_{h}$ and $b_{h}$ respectively.

Since $\angle a_{h} v b_{h}<\frac{2 \pi}{k}, k \geq 6$, we have

$$
\angle v b_{h} z=\frac{\pi}{2}<\pi-\angle a_{h} v b_{h}=\angle v b_{h} c_{h+1} .
$$

Similarly, $\angle v a_{h} z<\angle v a_{h} c_{h+1}$. This means $\widehat{a_{h} b_{h}}$ is inside $\triangle a_{h} b_{h} c_{h+1}$. The remaining of the proof directly follows from the proof for the case of $\triangle a_{i} b_{i} c_{i+1}$.

\section{Extended Yao-Yao Graph}

Using the space partition discussed in Section VI-B, we present our method to locally build a sparse network topology with bounded degree for heterogeneous wireless ad hoc network. Here we assume that $\gamma=\max _{v \in V} \gamma_{v}$ is bounded, where $\gamma_{v}=\max _{w \in I(v)} \frac{r_{v}}{r_{w}}$, and $I(v)=\left\{w \mid \overrightarrow{w v} \in \overrightarrow{E Y G}_{k}(M G)\right\}$.

\section{Algorithm 4: Constructing-EYY}

1. Each node finds the incident edges in the Extended Yao graph $\overrightarrow{E Y G}_{k}(M G)$, as described in Algorithm 3 .

2 . Each node $v$ partitions the $k$ cones centered at $v$ using the partitioning method described in Method 1. Notice that for partitioning, node $v$ uses parameter $\gamma_{v}$ in Method 1, which can be easily calculated from local information. Figure 5 (a) illustrates such a partition.

3. Each node $v$ chooses a node $u$ from each generated region so that the link $\overrightarrow{u v}$ has the smallest $I D(u v)$ among all directed links toward to $v$ computed in the first step in the partition. Figure 5 (b) illustrates such a selection of incoming links.

4. Finally, for each link $u v$ selected by $v$, node $v$ informs node $u$ of keeping link $u v$.

The union of all chosen links is the final network topology, denoted by $\overrightarrow{E Y Y}_{k}(M G)$. We call it extended Yao-Yao graph. Let $E Y Y_{k}(M G)$ be the undirected graph by ignoring the direction of each link in $\overrightarrow{E Y Y}_{k}(M G)$.

Theorem 7: The out-degree of each node $v$ in $\overrightarrow{E Y Y}_{k}(M G)$, $k \geq 6$, is bounded by $k$ and the in-degree is bounded by $\left(3\left\lceil\log _{2} \gamma_{v}\right\rceil+2\right) k$, where $\gamma_{v}=\max _{w \in I(v)}\left(\frac{r_{v}}{r_{w}}\right)$.

Proof: It is obvious that the out-degree of a node $v$ is bounded by $k$ because the out-degree bound of $\overrightarrow{E Y G}_{k}(M G)$ is $k$ and this algorithm does not add any directed link.

For the in-degree bound, as shown in Figure 2 (b), obviously, the number of triangle regions in each cone is $3 h-2$. Remember that $2^{h-2}<\gamma_{v} \leq 2^{h-1}$, which implies $h=1+\left\lceil\log _{2} \gamma_{v}\right\rceil$. Thus, considering the cap region also, the in-degree of node $v$ is at most $\left(3\left\lceil\log _{2} \gamma_{v}\right\rceil+2\right) k$.
Let $\gamma=\max _{v} \gamma_{v}$. Obviously, the maximum node degree in graph $E Y Y_{k}(M G)$ is bounded by $\left(3\left\lceil\log _{2} \gamma\right\rceil+3\right) k$.

Notice that the extended Yao-Yao graph $E Y Y_{k}(M G)$ is a subgraph of the extended Yao graph $E Y G_{k}(M G)$, thus, there are at most $k \cdot n$ edges in $E Y Y_{k}(M G)$. Thus, the total communications of Algorithm 4 is at most $O(k \cdot n)$, where each message has $O(\log n)$ bits. It is interesting to see that the communication complexity does not depend on $\gamma$ at all.

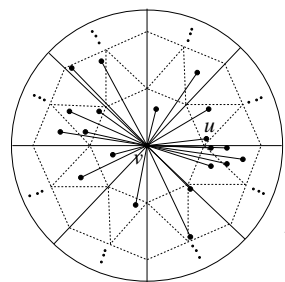

(a)

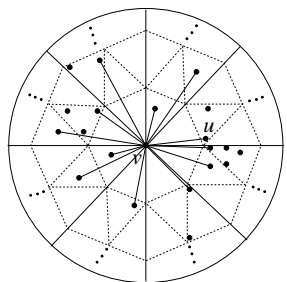

(b)

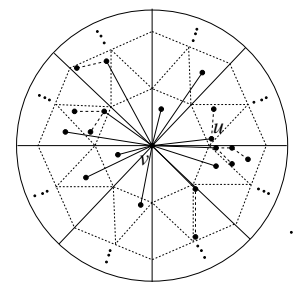

(c)
Fig. 5. (a) In $E Y G_{k}(M G)$, star formed by links toward to $v$. (b) Node $v$ chooses the shortest link in $E Y G_{k}(M G)$ toward itself from each region to produce $E Y Y_{k}(M G)$. (c) The sink structure at $v$ in $E Y Y_{k}^{*}(M G)$.

Theorem 8: The graph $E Y Y_{k}(M G), k \geq 6$, is connected if $\mathrm{MG}$ is connected .

Proof: Notice that it is sufficient to show that there is a path from $u$ to $v$ for any two nodes with $u v \in M G$. Remember the graph $E Y G_{k}(M G)$ is connected, therefore, we only have to show that $\forall u v \in E Y G_{k}(M G)$, there is a path connecting $u$ and $v$ in $E Y Y_{k}(M G)$. We prove this claim by induction on the ranks of all links in $E Y G_{k}(M G)$.

If the link $u v$ has the smallest rank among all links of $E Y G_{k}(M G)$, then $u v$ will obviously survive after the second step. So the claim is true for the smallest rank.

Assume that the claim is true for all links in $E Y G_{k}(M G)$ with rank at most $r$. Then consider a link $u v$ in $E Y G_{k}(V)$ with $\operatorname{rank}(u v)=r+1$ in $E Y G_{k}(M G)$. If $u v$ survives in Algorithm 4, then the claim holds. Otherwise, assume that $r_{u}<r_{v}$. Then directed edge $v u$ cannot belong to $\overrightarrow{E Y G}_{k}(M G)$ from Algorithm 3. Thus, directed edge $u v$ is in $\overrightarrow{E Y G}_{k}(M G)$. In Algorithm 4, directed edge $u v$ can only be removed by node $v$ due to the existence of another directed link $w v$ with a smaller identity and $w$ is in the same region as $u$. In addition, the angle $\angle w v u$ is less than $\theta=\frac{2 \pi}{k}(k \geq 6)$. Therefore we have $\|w u\|<\|u v\|$. Notice that here $w u$ is guaranteed to be a link in MG, but it is not guaranteed to be in $E Y G_{k}(M G)$. We then prove by induction that there is a path connecting $w$ and $u$ in $E Y Y_{k}(M G)$. Assume $r_{w} \leq r_{u}$. The scenario $r_{w}>r_{u}$ can be proved similarly. There are two cases here.

Case 1: the link $w u$ is in $E Y G_{k}(M G)$. Notice that rank of $w u$ is less than the rank of $u v$. Then by induction, there is a path $w \leadsto u$ connecting $w$ and $u$ in $E Y Y_{k}(M G)$. Consequently, there is a path (concatenation of the undirected path $w \leadsto u$ and the link $w v$ ) between $u$ and $v$.

Case 2: the link $w u$ is not in $E Y G_{k}(M G)$. Then, from proof of Theorem 4, we know that there is a path $\Pi_{E Y G_{k}}(w, u)=$ $q_{1} q_{2} \cdots q_{m}$ from $w$ to $u$ in $E Y G_{k}(M G)$, where $q_{1}=w$ and $q_{m}=u$. Additionally, we can show that each link $q_{i} q_{i+1}, 1 \leq$ $i<m$, has a smaller rank than $w u$, which is at most $r$. Here 900 $\operatorname{rank}\left(q_{1} q_{2}=w q_{2}\right)<\operatorname{rank}(w, u)$ because the selection method 
in Algorithm 3. And $\operatorname{rank}\left(q_{i} q_{i+1}\right)<\operatorname{rank}(w, u), 1<i<m$, because

$$
\left\|q_{i} q_{i+1}\right\| \leq\left\|q_{i} u\right\|<\left\|q_{i-1} u\right\|<\cdots<\left\|q_{1} u\right\|=\|w u\| .
$$

Then, by induction, for each link $q_{i} q_{i+1}$, there is a path $q_{i}$ $q_{i+1}$ survived in $E Y Y_{k}(M G)$ after Algorithm 4. The concatenation of all such paths $q_{i} \leadsto q_{i+1}, 1 \leq i<m$, and the link $w v$ forms a path from $u$ to $v$ in $E Y Y_{k}(M G)$.

Although $E Y Y_{k}(M G)$ is a connected structure, it is unknown whether it is a power or length spanner. We leave it as a future work.

\section{Extended Yao-Sink Graph}

In [3], [4], the authors applied the technique in [16] to construct a sparse network topology in UDG, Yao and sink graph, which has a bounded degree and a bounded stretch factor. The technique is to replace the directed star consisting of all links toward a node $v$ by a directed tree $T(v)$ with $v$ as the sink. Tree $T(v)$ is constructed recursively. To apply this technique on MG, we need extend it by a more sophisticated way. In the remainder of this section, we discuss how to locally construct a bounded degree structure with bounded power stretch factor for heterogeneous wireless ad hoc networks. Our method works as follows.

\section{Algorithm 5: Constructing-EYG*}

1. Each node finds the incident edges in the Extended Yao graph $\overrightarrow{E Y G}_{k}(M G)$, as described in Algorithm 3. Each node $v$ will have a set of incoming nodes $I(v)=\left\{u \mid \overrightarrow{u v} \in \overrightarrow{E Y G}_{k}(M G)\right\}$. 2. Each node $v$ partitions the $k$ cones centered at $v$ using the partitioning method described in Method 1. Notice that for partitioning, node $v$ uses parameter $\gamma_{v}$ in Method 1, which can be easily calculated from local information. Figure 5(a) illustrates such a partition.

3. Each node $v$ chooses a node $u$ from each region $\Omega$. Let $\Omega_{u}(v)$ be the region $\Omega$ partitioned by node $v$ with node $u$ inside, so that the link $u v$ has the smallest $I D(u v)$ among all links computed in the first step in the region $\Omega_{u}(v)$. In other words, in this step, it constructs $\overrightarrow{E Y Y_{k}(M G)}$.

4. For each region $\Omega_{u}(v)$ and the selected node $u$, let $S_{\Omega}(u)=$ $\left\{w \mid w \neq u, w \in \Omega_{u}(v) \cap I(v)\right\}$, i.e., the set of incoming neighbors of $v$ (other than $u$ ) in the same region as $u$. For each node $u$, node $v$ uses the following function $\operatorname{Tree}\left(u, S_{\Omega}(u)\right)$ (described in Algorithm 6) to build a tree $T(u)$ rooted at $u$. We call $T(u)$ a sink tree and call the union of all links chosen by node $v$ the sink structure at $v$. Figure 5(c) illustrates a sink structure at $v$, which is composed of all trees $T(u)$ for $u$ selected in the previous step.

5. Finally, node $v$ informs nodes $x$ and $y$ for each selected link $x y$ in the sink structure rooted at $v$.

The union of all chosen links is the final network topology, denoted by $E Y G_{k}^{*}(M G)$. We call such structure as the Extended Yao-Sink graph. Notice that, sink node $v$, not $u$, constructs the tree $T(u)$ and then informs the end-nodes of the selected links to keep such links if already exist or add such links otherwise.

Algorithm 6: Constructing-Tree $\operatorname{Tree}\left(u, S_{\Omega}(u)\right)$ 1. If $S_{\Omega}(u)$ is empty, then return.
2. Otherwise, partition the disk centered at $u$ by $k$ equal-sized cones: $\mathbb{C}_{1}(u), \mathbb{C}_{2}(u), \cdots, \mathbb{C}_{k}(u)$.

3. Find the node $w_{i} \in S_{\Omega}(u) \cap \mathbb{C}_{i}(u), 1 \leq i \leq k$, with the smallest $I D\left(w_{i} u\right)$, if there is any. Link $w_{i} u$ is added to $T\left(u, S_{\Omega}(u)\right)$ and node $w_{i}$ is removed from $S_{\Omega}(u)$.

4. For each node $w_{i}$, call $\operatorname{Tree}\left(w_{i}, S_{\Omega}(u) \cap \mathbb{C}_{i}(u)\right)$ and add the created edges to $T\left(u, S_{\Omega}(u)\right)$.

Notice that the above Algorithm 6 is only performed by a node $v$. We then prove that the constructed structure $\overrightarrow{E Y G}_{k}^{*}(M G)$ indeed has bounded degree (thus sparse), and is power efficient.

Theorem 9: The maximum node degree of the graph $\overrightarrow{E Y G}_{k}^{*}(M G)$ is at most $k^{2}+3 k+3 k \cdot\left\lceil\log _{2} \gamma\right\rceil$.

Proof: Initially, each node has at most $k$ out-degrees after constructing graph $E Y G_{k}(M G)$. In the algorithm, each node $v$ initiates only one sink structure, which will introduce at most $\left(3\left\lceil\log _{2} \gamma\right\rceil+2\right) \cdot k$ in-degrees. Additionally, each node $x$ will be involved in Algorithm 6 for at most $k$ sink trees (once for each directed link $x y \in E Y G_{k}(M G)$ ). For each sink tree involvement, Algorithm 6 assigns at most $k$ links incident on $x$. Thus, at most $k^{2}$ new degrees could be introduced here. Then the theorem follows.

Since the total number of edges is at most $\left(k^{2}+3 k+3 k\right.$. $\left.\left\lceil\log _{2} \gamma\right\rceil\right) \cdot n$, the total communication cost of our method is $O\left(\log _{2} \gamma \cdot n\right)$. Here each message has $O(\log n)$ bits.

Theorem 10: The length stretch factor of $E Y G_{k}^{*}(M G), k>$ 6 , is at most $\left(\frac{1}{1-2 \sin \left(\frac{\pi}{k}\right)}\right)^{2}$.

Proof: We have proved that $E Y G_{k}(M G)$ has length stretch factor at most $\frac{1}{1-2 \sin \left(\frac{\pi}{k}\right)}$. We thus have only to prove that, for each link $v w \in E Y G_{k}(M G)$, there is a path connecting them in $E Y G_{k}^{*}(M G)$ with length at most $\frac{1}{1-2 \sin \left(\frac{\pi}{k}\right)}\|v w\|$. If link $v w$ is kept in $E Y G_{k}^{*}(M G)$, then this is obvious. Otherwise, assume $r_{w} \leq r_{v}$, then directed link $w v$ belongs to $\overrightarrow{E Y G}_{k}(M G)$. Then, there must exist a node $u$ in the same region (partitioned by node $v$ ) as node $w$. Using the same argument as Theorem 4, we can prove that there is a path connecting $v$ and $w$ in $T(u)$ with length at most $\frac{1}{1-2 \sin \left(\frac{\pi}{k}\right)}\|v w\|$. It implies that the length stretch factor of $E Y G_{k}^{*}(M G)$ is at most $\left(\frac{1}{1-2 \sin \left(\frac{\pi}{k}\right)}\right)^{2}$.

Similarly, we have:

Theorem 11: The power stretch factor of the graph $E Y G_{k}^{*}(M G)$, $k>6$, is at most $\left(\frac{1}{1-\left(2 \sin \frac{\pi}{k}\right)^{\beta}}\right)^{2}$.

\section{Simulations}

In this section we measure the performance of the proposed heterogeneous network topologies by conducting extensive simulations. In our simulations, we randomly generate a set $V$ of $n$ wireless nodes with random transmission range for each node. We then construct the mutual inclusion communication graph $M G(V)$, and test the connectivity of $M G(V)$. If it is connected, we construct different localized topologies: $G G(M G), E G G(M G), R N G(M G), E R N G(M G)$, $E Y G_{k}(M G), E Y Y_{k}(M G)$ and $E Y G_{k}^{*}(M G)$. Then we measure the sparseness (the average node degree), the power efficiency and the communication cost of building these topologies. 291 In the simulation results presented here, the wireless nodes are 
distributed in a $400 m \times 400 m$ square field. Each wireless node has a transmission radius randomly selected from $[60 \mathrm{~m}, 260 \mathrm{~m}]$. The number of wireless nodes is $30 i$, where $i$ is varied from 1 to 10 . For each $1 \leq i \leq 10$, we randomly generate 100 sets of $30 i$ nodes. All structures proposed in this paper are generated for each set of nodes. The number of cones is set to 7 for $E Y G_{k}(M G), E Y Y_{k}(M G)$ and $E Y G_{k}^{*}(M G)$.

\section{A. Node Degree}

First of all, we want to test the sparseness of each network topology proposed in this paper. Notice that, we have theoretically proved that $R N G(M G)$ and $E R N G(M G)$ have at most $6 n$ links; $E Y G_{k}(M G)$ has at most $k \cdot n$ links, where $k \geq 7$ is the number of cones divided; $E Y Y_{k}(M G)$ also has at most $k \cdot n$ links since $E Y Y_{k}(M G) \subseteq E Y G_{k}(M G) ; E Y G_{k}^{*}(M G)$ also has at most $k \cdot n$ links since the sink structure for each node $u$ has exactly the number of links as the links toward $u$ in the directed structure $E Y G_{k}(M G)$. We do not know how many links $G G(M G)$ and $E G G(M G)$ could have.

Although almost all proposed structures are sparse theoretically, all of them could have unbounded node degree. The node degree of the wireless networks should not be too large. Otherwise a node with a large degree has to communicate with many nodes directly. This potentially increases the signal interference and the overhead at this node. Figure 6 (a) illustrates the average node degree of different topologies. Notice that graph $R N G(M G)$ always has the smallest average node degree in our simulations and structure $E Y G_{k}^{*}(M G)$ always has the largest average node degree. We also found that the average node degree becomes almost stable when the number of nodes increases, i.e., the network becomes denser.

Figure 6 (b), as proved in Theorem 9, confirms that the maximum node degree of Yao-based structure $E Y G_{k}^{*}(M G)$ is bounded by $3 k \cdot \log _{2} \gamma+k^{2}+3 k$, where $\gamma=\max _{u v \in M G} \frac{r_{u}}{r_{v}}$. The most upper curve in Figure 6 (b) represents the maximum $3 k \cdot \log _{2} \gamma$. This figure also shows that $E Y G_{k}(M G)$ generally will have a larger maximum node degree than $E Y G_{k}^{*}(M G)$ and $E Y Y_{k}(M G)$. It is interesting to see that the maximum degree of $E Y G_{k}^{*}(M G)$ and $E Y Y_{k}(M G)$ almost have the same curve when network density changes. Given the size of the network $n=30 i$, we take the average of the maximums of all 100 random networks with $n$ nodes we generated as the final maximum value for $n$ plotted here.

\section{B. Spanning ratio}

We proved that $G G(M G)$ and $E G G(M G)$ have power spanning ratio exactly one; $E Y G_{k}(M G)$ and $E Y G_{k}^{*}(M G)$ both have bounded length and power spanning ratios. Notice that $R N G(M G)$ and $E R N G(M G)$ could have power and length spanning ratios as large as $n-1$ for a network of $n$ nodes; and the length spanning ratios of $G G(M G)$ and $E G G(M G)$ could be $\sqrt{n-1}$ even when all nodes have the same transmission range. It is unknown whether $E Y Y_{k}(M G)$ has a bounded length or power spanning ratio even for wireless networks modelled by UDG. We then conduct extensive simulations to study how good these structures are for heterogeneous networks when the nodes' transmission ranges are randomly set.
Figure 6 (c) illustrates the length spanning ratio of these structures. As the theoretical results suggest, we found that $R N G(M G)$ has a much larger length spanning ratio compared with other structures. It is surprising to see that $E R N G(M G)$ also has a much smaller spanning ratio than $R N G(M G)$. We know that $E R N G(M G)$ has a smaller spanning ratio than $R N G(M G)$ since $E R N G(M G) \supseteq R N G(M G)$. Also notice that $E Y G_{k}(M G)$, as the theoretical results suggest, has the smallest spanning ratio among all structures proposed here.

For wireless ad hoc networks, we want to keep as less links as possible while still keep relatively power efficient paths for every pair of nodes. Figure 6 (d) illustrates the power spanning ratio of these structures. Here we assume that the power needed to support a link $u v$ is $\|u v\|^{2}$. As we expected, structures $G G(M G)$ and $E G G(M G)$ keep the most power efficient path for every pair of nodes, i.e., their power spanning ratios are exactly one. We found that all structures have power spanning ratio almost one, and again $R N G(M G)$ and $E R N G(M G)$ do have the largest power spanning ratios in our simulations.

\section{Communication Cost of Construction}

It is not difficult to see that $G G(M G), R N G(M G)$, and $E Y G_{k}(M G)$ can be constructed using only $n$ messages by assuming that each node can tell its neighbors its maximum transmission range, and its geometry position information in one single message. Each node $u$ can uniquely determine all the links $u v$ in these three structures after knowing all its one hop neighbors in $M G$. Structures $E Y G_{k}(M G)$, and $E Y G_{k}^{*}(M G)$ can be constructed using only $k \cdot n+n$ messages since the final

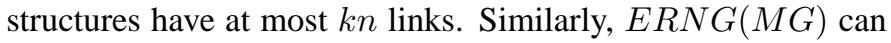
be constructed using at most $7 n$ messages. We do not know any theoretical bound about the number of messages needed to construct $E G G(M G)$ since each node $u$ has to inform its neighbors the links selected by $u$ for $E G G(M G)$. We measured the actual average number of messages needed to construct these structures. We only measure the average number of messages per wireless node for $E G G(M G), E R N G(M G), E Y G_{k}(M G)$, and $E Y G_{k}^{*}(M G)$ (since every node only has to spend one message for other three structures $G G(M G), R N G(M G$, and $\left.E Y G_{k}(M G)\right)$. Figure 7 illustrates the communication cost. We

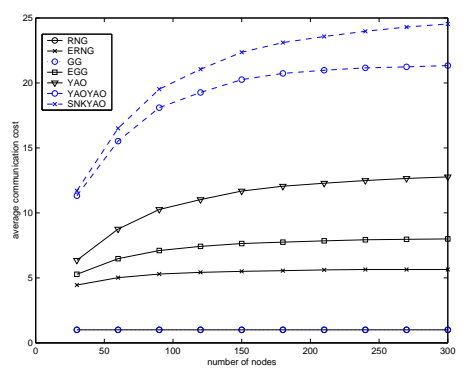

Fig. 7. Average communication cost of building different topologies.

found that structure $E Y G_{k}^{*}(M G)$ is the most expensive one to construct although it has the most favorable properties theoretically (bounded length, power spanning ratio and bounded node degree). Constructing $E Y G_{k}^{*}(M G)$ is almost as expensive as $292^{\text {constructing }} E Y G_{k}(M G)$. 


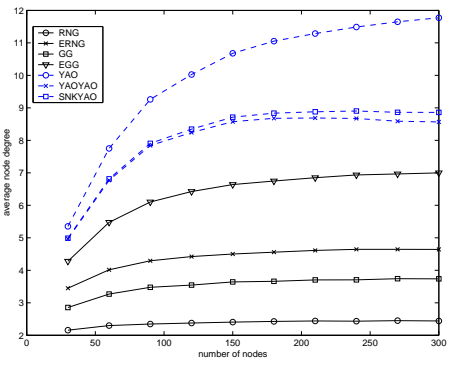

(a)

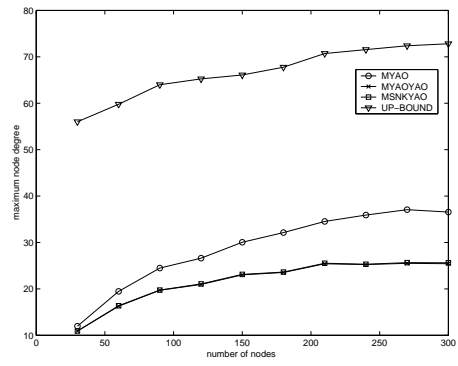

(b)

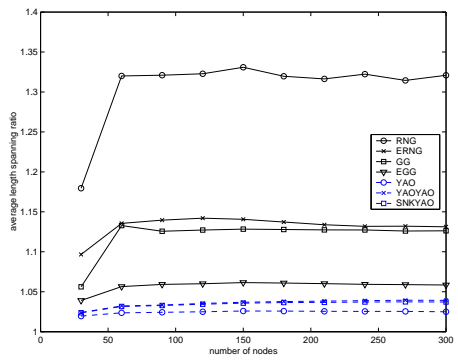

(c)

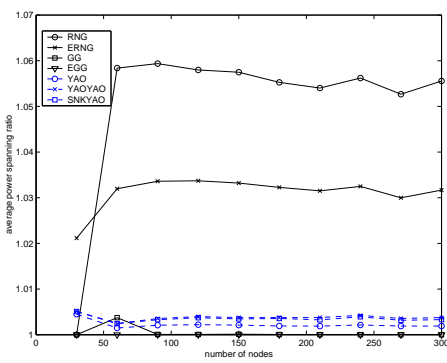

(d)

Fig. 6. (a) Average node degree of different topologies. (b) Maximum node degree of Yao-based structures. (c) Average length spanning ratio of different topologies. (d) Average power spanning ratio of different topologies.

\section{CONCLUSION}

In this paper, we studied topology control in heterogeneous wireless ad hoc networks, where each mobile host has different maximum transmission power and two nodes are connected iff they are within the maximum transmission range of each other. We presented several strategies for all wireless nodes self-maintaining sparse and power efficient topologies in heterogeneous network environment with low communication cost. All structures $G G(M G), R N G(M G), E Y G_{k}(M G)$, $E Y Y_{k}(M G)$, and $E Y G_{k}^{*}(M G)$ are connected if $\mathrm{MG}$ is connected, while $E Y G_{k}(M G)$ and $E Y G_{k}^{*}(M G)$ have constant bounded power and length stretch factors. Additionally, we showed that $E Y Y_{k}(M G)$ and $E Y G_{k}^{*}(M G)$ have bounded node degrees $O\left(\log _{2} \gamma\right)$, where $\gamma=\max _{v \in V} \max _{w \in I(v)}\left(\frac{r_{v}}{r_{w}}\right)$. In the worst cast any connected graph will have degree at least $O\left(\log _{2} \gamma\right)$ for heterogeneous wireless ad hoc networks. In other words, the structures constructed by our method are almost optimum. Our algorithms are all localized and have communication cost at most $O(n)$, where each message has $O(\log n)$ bits.

It remains an open problem whether graph $E Y Y_{k}(M G)$ is a length or power spanner. It is also unknown how many links $G G(M G)$ could have in the worst case although we knew that it is definitely less than $O\left(n^{8 / 5} \log _{2} \gamma\right)$. Some other future works are what are the conditions that we can build a structure with some other properties for MG, such as planar or low weight. Notice that it is easy to show we cannot build a planar topology for an arbitrary heterogeneous wireless ad hoc network.

\section{REFERENCES}

[1] L. Hu, "Topology control for multihop packet radio networks," IEEE Trans. Communications, vol. 41, no. 10, 1993.

[2] L. Li, J. Y. Halpern, P. Bahl, Y.-M. Wang, and R. Wattenhofer, "Analysis of a cone-based distributed topology control algorithms for wireless multihop networks," in ACM PODC, 2001.

[3] X.-Y. Li, P.-J. Wan, and Y. Wang, "Power efficient and sparse spanner for wireless ad hoc networks," in IEEE ICCCN, 2001, pp. 564-567.

[4] X.-Y. Li, P.-J. Wan, Y. Wang, and O. Frieder, "Sparse power efficient topology for wireless networks," in IEEE Hawaii Int. Conf. on System Sciences (HICSS), 2002.

[5] R. Ramanathan and R. Rosales-Hain, "Topology control of multihop wireless networks using transmit power adjustment," in IEEE INFOCOM, 2000.

[6] R. Wattenhofer, L. Li, P. Bahl, and Y.-M. Wang, "Distributed topology control for wireless multihop ad-hoc networks," in IEEE INFOCOM, 2001.

[7] L. Barrire, P. Fraigniaud, and L Narayanan, "Robust position-based routing in wireless ad hoc networks with unstable transmission ranges," in ACM DialM-POMC, 2001.
[8] F. Kuhn and A. Zollinger, "Ad-hoc networks beyond unit disk graphs," in ACM DialM-POMC, 2003.

[9] G. T. Toussaint, "The relative neighborhood graph of a finite planar set," Pattern Recognition, vol. 12, no. 4, pp. 261-268, 1980.

[10] K.R. Gabriel and R.R. Sokal, "A new statistical approach to geographic variation analysis," Systematic Zoology, vol. 18, pp. 259-278, 1969.

[11] X.-Y. Li, G. Calinescu, and P.-J. Wan, "Distributed construction of planar spanner and routing for ad hoc wireless networks," in IEEE INFOCOM, 2002.

[12] A. C.-C. Yao, "On constructing minimum spanning trees in k-dimensional spaces and related problems," SIAM J. Computing, vol. 11, pp. 721-736, 1982.

[13] T. Lukovszki, New Results on Geometric Spanners and Their Applications, Ph.D. thesis, University of Paderborn, 1999.

[14] W.-Z. Song, Y. Wang, and X.-Y. Li, "Efficient topology control for wireless networks with non-uniform transmission ranges," 2003, ACM WINET, accepted for publication. To appear.

[15] N. Li, J. C. Hou, and L. Sha, "Topology control in heterogeneous wireless networks: Problems and solutions," in IEEE INFOCOM, 2004

[16] S. Arya, G. Das, D. Mount, J. Salowe, and M. Smid, "Euclidean spanners: short, thin, and lanky," in Proc. 27th ACM STOC, 1995, pp. 489-498.

[17] Y. Wang and X.-Y. Li, "Localized construction of bounded degree planar spanner for wireless networks," in ACM DialM-POMC, 2003.

[18] W.-Z. Song, Y. Wang, X.-Y. Li, and O. Frieder, "Localized algorithms for energy efficient topology in wireless ad hoc networks," in ACM MobiHoc, 2004.

[19] S. Kapoor and X.-Y. Li, "Geometric proximity graphs," in WADS, 2003. 